\title{
Genetically optimised placement of piezoelectric sensor arrays: linear and nonlinear transient analysis
}

\author{
J. N. Rao, S. Lentzen \& R. Schmidt \\ Institute of General Mechanics, RWTH Aachen University, Germany
}

\begin{abstract}
In modern structural control the application of discrete modal sensor arrays is a commonly used technique to obtain the modal state vectors. In this paper, a genetic algorithm is used to find the optimal positions to place modal sensor arrays on simple structures such as beams, plates and shells. The performance criterion is taken as the steady state observability Grammian of the system and includes spillover prevention as well. The performance of optimally placed modal sensors in the linear range is discussed. The variation in the performance of these modal sensors in the nonlinear range is investigated.

Keywords: geometrical nonlinearity, modal sensors, Lyapunov equation, genetic algorithm.
\end{abstract}

\section{Introduction}

There has been consistent research on smart materials and structures for the last two decades. Geometrically linear theories and numerical methods have been developed by many authors, e.g. Crawley and de Luis [1], Tzou and Tseng [2]. Considerably less work can be found in the area of geometrically nonlinear modelling of smart structures. Structural nonlinearity has been taken into account for interlaminar stress analysis by Icardi and Di Sciuva [3], for large deflection shape control in Yi et al. [4], Mukherjee and Chaudari [5] and Lentzen and Schmidt [6]. Active buckling control and post-buckling analysis has been done by Krishna and Mei [7] and Chandrashekhara and Bhatia [8].

Piezothermoelastic analysis including nonlinearity is discussed in Tzou et al. [9] and Pai et al. [10]. 
In order to implement structural vibration control in a modern fashion, one should be able to sense the modal amplitudes. Several possibilities of modal sensing can be found in the literature. Clark and Burke [11], Lee and Moon [12], Gawronski [13] are some of the earlier researchers, who worked on modal sensors. The optimal locations of sensors in intelligent structures is an important issue in the research of present day, since an arbitrary decision can degrade their performance. In order to find the optimal positions of these modal sensors, genetic algorithms (GAs) can be used as a searching method. Some of the earlier successful attempts can be found in the works of Sadri et al. [14], Han and Lee [15], where GA is used to find optimal places for both sensors as well as actuators.

In the present paper discrete array sensors are used to filter the modal amplitudes. To find the optimal locations of modal sensors the observability of the system should be investigated to obtain the best performance with the least number of sensors. The performance criterion for the selection of the sensor positions is taken as the steady state observability Grammian of the system. The steady state observability Grammian is obtained by solving the Lyapunov equation. The performance of genetically optimised sensor arrays in the linear range is discussed, and the influence of structural nonlinearity is investigated.

\section{Structural model}

The FE analysis of the structural response is performed using the theory of composite laminated shells given by Schmidt and Reddy [16]. The geometrically nonlinear strain displacement relations are based on the Reissner-Mindlin hypothesis and are valid for small strains and moderate rotations of the midsurface normals [16]

$$
\begin{aligned}
& \varepsilon_{\alpha \beta}=\stackrel{0}{\varepsilon}_{\alpha \beta}+\Theta^{3} \stackrel{1}{\varepsilon}_{\alpha \beta}+\left(\Theta^{3}\right)^{2} \stackrel{2}{\varepsilon}_{\alpha \beta} \\
& \varepsilon_{\alpha 3}=\stackrel{0}{\varepsilon}_{\alpha 3}+\Theta^{3} \stackrel{1}{\varepsilon}_{\alpha 3} \\
& \varepsilon_{33}=0
\end{aligned}
$$

with

$$
\begin{gathered}
\stackrel{0}{\varepsilon}_{\alpha \beta}=\stackrel{0}{\theta}_{\alpha \beta}+\frac{1}{2} \stackrel{0}{\varphi}_{\alpha} \stackrel{0}{\varphi}_{\beta} \\
\stackrel{1}{\varepsilon}_{\alpha \beta}=\frac{1}{2}\left(\stackrel{1}{v}_{\alpha \mid \beta}+\stackrel{1}{v}_{\beta \mid \alpha}-b_{\alpha}^{\lambda} \stackrel{0}{\varphi}_{\lambda \beta}-b_{\beta}^{\lambda} \stackrel{0}{\varphi}_{\lambda \alpha}+\stackrel{0}{\varphi}_{\alpha} b_{\beta}^{\lambda} \stackrel{1}{v}_{\lambda}+\stackrel{0}{\varphi}_{\beta} b_{\alpha}^{\lambda} \stackrel{1}{v}_{\lambda}\right), \\
\stackrel{2}{\varepsilon}_{\alpha \beta}=\frac{1}{2}\left(b_{\alpha}^{\lambda} b_{\beta}^{\kappa} \stackrel{1}{v}_{\lambda} \stackrel{1}{v}_{\kappa}-b_{\alpha}^{\lambda} \stackrel{1}{v}_{\lambda \mid \beta}-b_{\beta}^{\lambda} \stackrel{1}{v}_{\lambda \mid \alpha}\right) \\
\stackrel{0}{\varepsilon}_{\alpha 3}=\frac{1}{2}\left(\stackrel{0}{\varphi}_{\alpha}+\stackrel{1}{v}_{\alpha}+\stackrel{1}{v}^{\lambda} \stackrel{0}{\varphi}_{\lambda \alpha}\right) \text { and } \stackrel{1}{\varepsilon_{\alpha 3}}=\frac{1}{2} \stackrel{1}{v}{ }^{\lambda} \stackrel{1}{v}_{\lambda \mid \alpha} .
\end{gathered}
$$

The following abbreviations were used:

$$
\stackrel{0}{\theta}_{\alpha \beta}=\frac{1}{2}\left(\stackrel{0}{v}_{\alpha \mid \beta}+\stackrel{0}{v}_{\beta \mid \alpha}\right)-b_{\alpha \beta} \stackrel{0}{v}_{3},
$$




$$
\stackrel{0}{\varphi}_{\alpha \beta}=\stackrel{0}{v}_{\alpha \mid \beta}-b_{\alpha \beta} \stackrel{0}{v}_{3} \quad \text { and } \quad \stackrel{0}{\varphi}_{\alpha}=\stackrel{0}{v}_{3, \alpha}+b_{\alpha}^{\lambda} \stackrel{0}{v}_{\lambda}
$$

The internal and external virtual work are evaluated in a total Lagrangian fashion. The second Piola-Kirchhoff stress and Green-Lagrange strain are chosen to express the mechanical part of the internal virtual work. Consequently, the electric variables are defined as referring to the initial undeformed configuration [17].

\section{Performance criterion}

The performance criterion to optimise the modal sensor positions requires modal voltages. The linear equations of motion of a laminated composite structure with embedded piezoelectric layers can be written as

$$
\left[\begin{array}{cc}
\mathbb{M} & 0 \\
0 & 0
\end{array}\right]\left\{\begin{array}{l}
\ddot{q} \\
0
\end{array}\right\}+\left[\begin{array}{ll}
\mathbb{K}_{q q} & \mathbb{K}_{q \phi} \\
\mathbb{K}_{\phi q} & \mathbb{K}_{\phi \phi}
\end{array}\right]\left\{\begin{array}{l}
q \\
\phi
\end{array}\right\}=\left\{\begin{array}{c}
\mathbb{F}_{e} \\
\mathbb{Q}_{e}
\end{array}\right\}
$$

where the above matrices are found in Lentzen et al. [17]. There, $\mathbb{M}$ is the mass matrix, $\mathbb{K}_{q q}$ is the linear elastic stiffness matrix, $\mathbb{K}_{q \phi}=\mathbb{K}_{\phi q}$ are the linear electromechanical coupling matrices and $\mathbb{K}_{\phi \phi}$ is the piezoelectric capacitance matrix. The electromechanical response of the structure is denoted by $\{q, \phi\}$, where $q$ and $\phi$ are the system's generalised displacements and sensor voltages, respectively. The externally applied forces $\left(\mathbb{F}_{e}\right)$ and charges $\left(\mathbb{Q}_{e}\right)$ are denoted by the right subscript $e$.

After static condensation of the electric variables, equation (2) can be written in modal form as

$$
\ddot{p}_{j}+\omega_{j}^{2} p_{j}=\sum_{L=1}^{R} B_{j L}^{\prime} V_{L}
$$

where $\ddot{p}_{j}, \dot{p}_{j}$ and $p$ represent the $j^{\text {th }}$ modal acceleration, velocity and displacement, respectively. The natural frequency of the $j^{\text {th }}$ mode is denoted by $\omega_{j}$. The piezo sensor voltages can be represented as

$$
\Phi_{L}=\sum_{j=1}^{\infty} C_{j L}^{\prime} p_{j}
$$

Here the induced voltage of the $L^{\text {th }}$ sensor and the control voltage at the $L^{\text {th }}$ actuator are denoted by $\Phi_{L}$ and $V_{L}$, respectively. The sensing constant of the $L^{\text {th }}$ sensor due to unit modal displacement of the $j^{\text {th }}$ mode is denoted by $C_{j L}^{\prime}$ and the $j^{\text {th }}$ modal actuation constant due to unit applied voltage of the $L^{\text {th }}$ actuator is denoted by $B_{j L}^{\prime}$.

In order to address the observability problem one should convert the standard FE equations into state space equations as follows [15]:

$$
\begin{gathered}
\dot{x}=[\mathbf{A}]\{x\}+[\mathbf{B}]\{u\} \\
y=[\mathbf{C}]\{x\}
\end{gathered}
$$


where

$$
\begin{aligned}
& x=\left[\begin{array}{lllll}
\dot{p_{1}} & \omega_{1} p_{1} & \ldots & \dot{p_{n}} & \omega_{n} p_{n}
\end{array}\right]^{T} \\
& \mathbf{A}=\operatorname{diag}\left(\mathbf{A}_{i}\right), \quad \mathbf{A}_{i}=\left[\begin{array}{cc}
0 & -\omega_{i} \\
\omega_{i} & 0
\end{array}\right] \\
& u=\left[\begin{array}{c}
V_{1} \\
\vdots \\
V_{R}
\end{array}\right], \quad \mathbf{B}=\left[\begin{array}{ccc}
B_{11}^{\prime} & \cdots & B_{1 R}^{\prime} \\
0 & \cdots & 0 \\
\vdots & \vdots & \vdots \\
B_{n 1}^{\prime} & \cdots & B_{n R}^{\prime} \\
0 & \cdots & 0
\end{array}\right] \\
& y=\left[\begin{array}{c}
\Phi_{1} \\
\vdots \\
\Phi_{s}
\end{array}\right] \text { and } \mathbf{C}=\left[\begin{array}{ccc}
0 & \cdots & 0 \\
\omega_{1}^{-1} C_{11}^{\prime} & \cdots & \omega_{1}^{-1} C_{1 S}^{\prime} \\
\vdots & \vdots & \vdots \\
0 & \cdots & 0 \\
\omega_{n}^{-1} C_{n 1}^{\prime} & \cdots & \omega_{n}^{-1} C_{n S}^{\prime}
\end{array}\right]^{T}
\end{aligned}
$$

A linear time invariant system $(\mathbf{A}, \mathbf{B}, \mathbf{C})$, with $s$ outputs is completely observable, if any of the following conditions are satisfied [19].

1. The $(s n \times n)$ observability matrix [O] has rank $n$, where

$$
[\mathbf{O}]=\left[\begin{array}{lllll}
{[\mathbf{C}]} & {[\mathbf{C}][\mathbf{A}]} & {[\mathbf{C}][\mathbf{A}]^{2}} & \cdots & {[\mathbf{C}][\mathbf{A}]^{n-1}}
\end{array}\right]^{T}
$$

2. The observability Grammian $\left[W_{o}\right]$ is full-rank

$$
\left[W_{o}\right]=\int_{0}^{\infty} e^{[\mathbf{A}] t}[\mathbf{C}][\mathbf{C}]^{T} e^{[\mathbf{A}]^{T} t} d t
$$

In fact $\left[W_{o}\right]$ is the solution of the Lyapunov equation:

$$
[\mathbf{A}]\left[W_{o}\right]+\left[W_{o}\right][\mathbf{A}]^{T}+[\mathbf{C}][\mathbf{C}]^{T}=0
$$

The observability test based on the rank is binary in nature (i.e. it tells us whether the system is observable or not). Additionally the extent of observability is required. Therefore, in the present work the objective function proposed by Hac and Liu [18] is used, given by:

$$
J=\left(\sum_{j=1}^{2 n_{o}} \lambda_{j}\right) \sqrt[2 n_{o}]{\prod_{j=1}^{2 n_{o}}\left(\lambda_{j}\right)}-\gamma\left(\sum_{j=2 n_{o}+1}^{2\left(n_{o}+n_{r}\right)} \lambda_{j}\right) \sqrt[2 n_{r}]{\prod_{j=2 n_{o}+1}^{2\left(n_{o}+n_{r}\right)}\left(\lambda_{j}\right)},
$$

where $n_{o}$ and $n_{r}$ are the number of observed modes and residual modes, respectively. For sensor optimal places, $\lambda_{j}$ is the eigenvalue of the steady state observability Grammian (i.e. $\left.\left[W_{o}\right]\right)$. Note that in the objective function (i.e. Eq. 11) a 
product term is included to account for least controlled modes. If any of the modes is least controlled then the total objective function value goes to zero.

\section{Genetic algorithm}

Genetic algorithms (GAs) are random search techniques based on the mechanics of natural selection and genetics. Genetic algorithms are used to explore the global extremum of the given linear or nonlinear function. Although randomised, genetic algorithms can efficiently explore the new generation with better fitness.

The GA is used to maximise $J$ (i.e. Eq. (11)) for a given number of sensors.

\subsection{Algorithm}

1. Create a random initial population of sensors.

2. Evaluate each member of the current population by computing its fitness value (i.e. $\mathrm{J}$ as given in Eq. (11) ), and select parents based on their fitness value.

3. Children are produced by mating a randomly selected pair of parents at a randomly selected site, known as crossover and by making random changes to a single parent, known as mutation.

4. Replace the current population with the children from the new generation.

5. Repeat the algorithm for a prescribed number of generations.

\subsection{Modal sensors}

The principle of discrete modal sensor arrays is depicted in Figure 1. By choosing the gains $\alpha_{i}$ in a particular way, the modal sensor will respond only to the mode $j$. The gains are obtained by solving the orthogonal system of equations

$$
[G]\left\{\alpha_{j}\right\}=\left\{e_{j}\right\},
$$

where $[G]=g_{k i}$, which is the modal voltage of the $k^{t h}$ sensor due to the unit modal displacement of mode $i,\left\{\alpha_{j}\right\}$ are the linear gains of all sensors to sense mode $j$ and $\left\{e_{j}\right\}$ is the unit vector in the direction $j$.

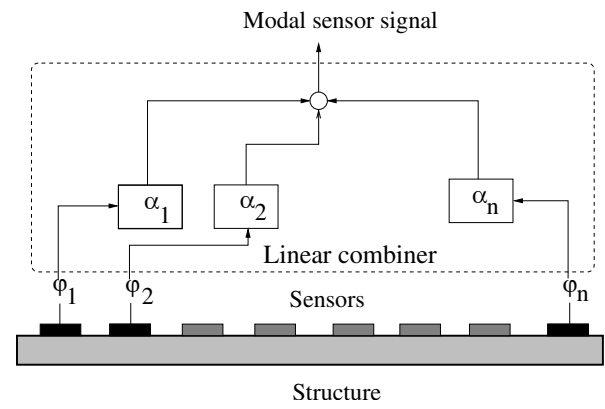

Figure 1: Principle of the discrete modal sensor array. 


\section{Numerical results}

\subsection{Clamped plate}

A plate, with the dimensions $[110 \times 110 \times 1] \mathrm{mm}$ as shown in Figure 2, is taken as a numerical example. The plate consists of aluminium and is meshed with a [11x11] grid. The material properties of the aluminium and PVDF layers are displayed in Table 1. The position of the four patches has to be optimised in order to observe the first four modes. The GA parameters used in the analysis are population size (30), crossover probability (50\%), mutation probability (10\%) and number of generations (40). After application of GA, the optimal sensor positions for the plate are obtained as depicted in Figure 2. The first four natural frequencies are calculated as $728 \mathrm{~Hz}, 1486 \mathrm{~Hz}, 1486 \mathrm{~Hz}$ and $2193 \mathrm{~Hz}$.

Table 1: Aluminium and piezo material properties.

\begin{tabular}{|c|c|c|}
\hline & Aluminium & PVDF \\
\hline$E[G p a]$ & 70 & 2 \\
$\nu[-]$ & 0.3 & 0.3 \\
$\rho\left[k g / \mathrm{m}^{3}\right]$ & 2700 & 2800 \\
$d_{31}[\mathrm{~m} / \mathrm{V}]$ & - & $2.2 \cdot 10^{-10}$ \\
$d_{33}[\mathrm{~m} / \mathrm{V}]$ & - & $1.062 \cdot 10^{-11}$ \\
\hline
\end{tabular}

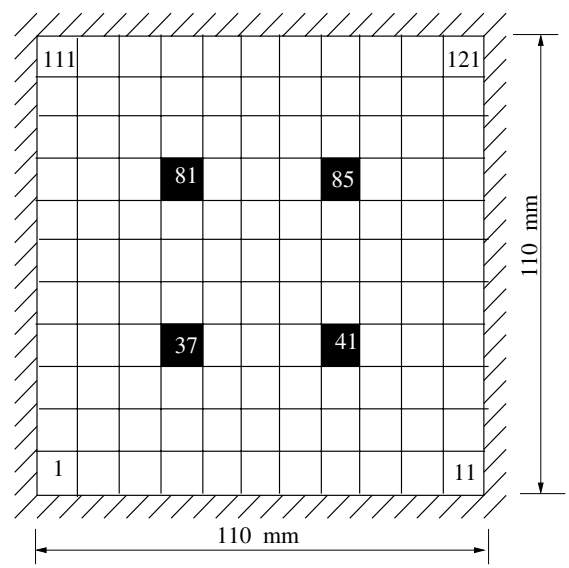

Figure 2: Optimised configuration.

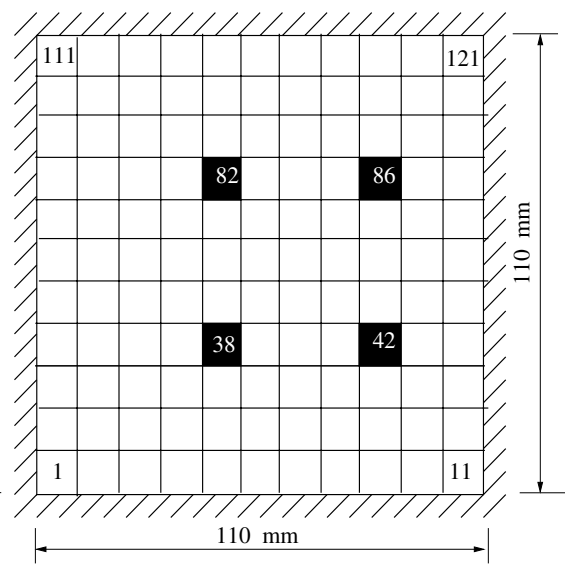

Figure 3: Arbitrary configuration.

In order to examine the effectiveness of the modal sensors, the transient modal sensor signals have to be investigated. One of the popular explicit time integration techniques (i.e. central difference method) is used to integrate the equations (2) 
in the time domain. As an initial displacement field, the superposition of the first four modal displacements is prescribed, with the respective modal amplitudes of $0.01,0.007,0.007$ and 0.003 resulting in a mid-point deflection of $0.3616 \mathrm{~mm}$. Figure 3 shows an arbitrary configuration of sensors that is considered in the present work for comparison purpose. Figures 4-7 compare the results obtained with the optimised and the arbitrary configuration. It can be observed in Figures 4-7 that the modal sensor signals for the optimal sensor configuration are correct and are barely aliased with those of higher modes. In case of the arbitrary sensor configuration the modal sensor signals are strongly aliased with those of higher modes. Figures 8-9 show the modal signals for both optimal sensor configuration and arbitrary sensor configuration in the nonlinear case. It can be concluded that the induced membrane stresses which are not considered in the linear and modal analysis are the main cause for the failure of modal sensor arrays in the nonlinear range of deformations.

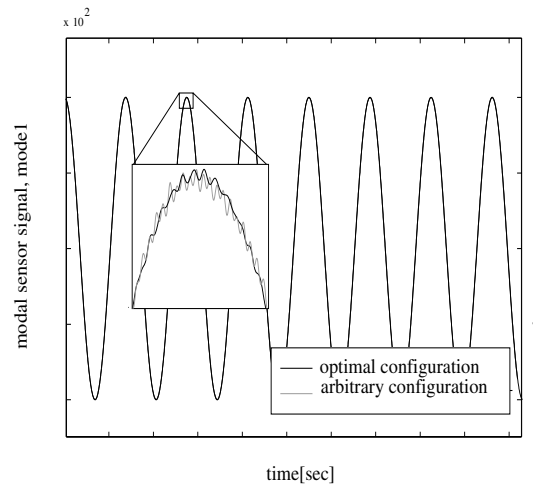

Figure 4: First mode signal.

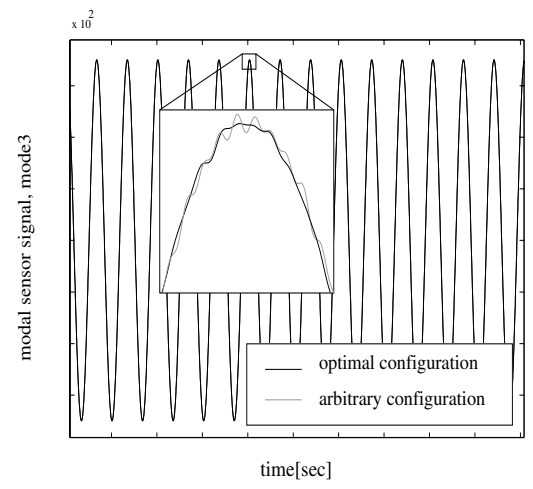

Figure 6: Third mode signal.

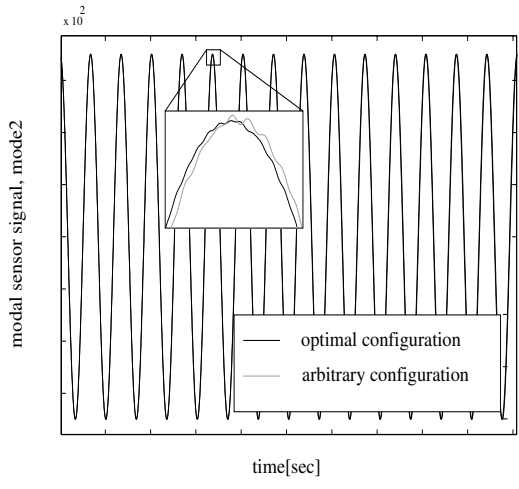

Figure 5: Second mode signal.

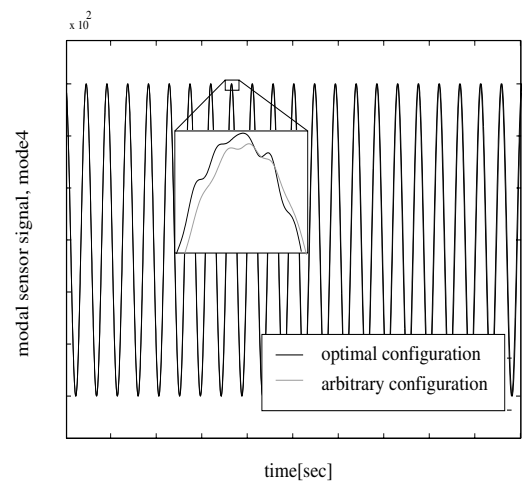

Figure 7: Fourth mode signal. 


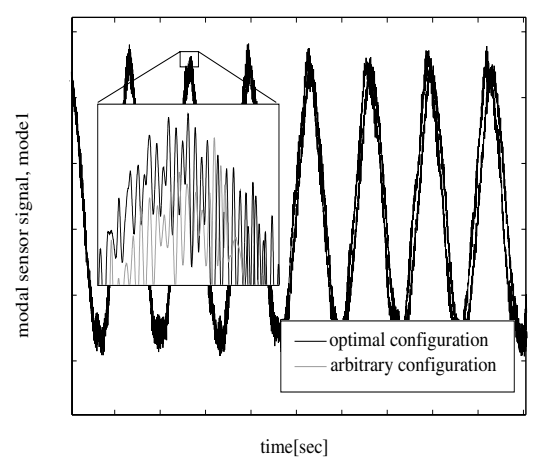

Figure 8: Nonlinear first mode signal.

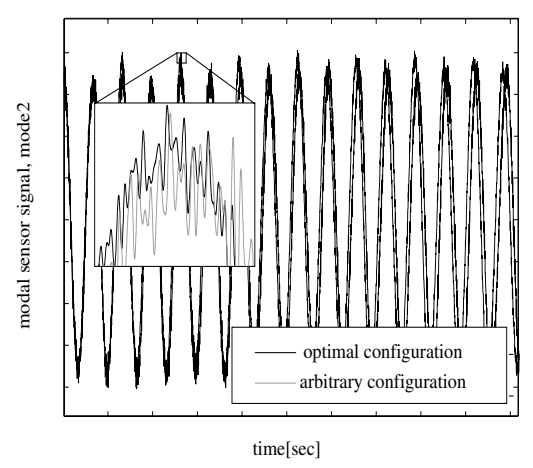

Figure 9: Nonlinear second mode signal.

\section{Conclusions}

In the present work, GA is used to find optimal placement for modal sensors. Transient analysis is performed with linear and nonlinear FE, based on first-order shear deformation moderate rotation theory. By numerical example it is shown that the principle of modal sensor arrays yields good results for the genetically optimally placed sensor patches in the range of small displacements. In the geometrically nonlinear case, it is found that the induced membrane stresses are the prime cause for the failure of modal sensor arrays.

\section{References}

[1] E. F. Crawley and J. de Luis, Use of piezoelectric actuators as elements of intelligent structures. AIAA Journal, 25, 1373-1385, 1987.

[2] H. S. Tzou and C. I. Tseng, Distributed piezoelectric sensor/actuator design for dynamic measurement/control of distributed parameter systems: a piezoelectric finite element approach. J. Sound Vib., 138, 17-34, 1990.

[3] U. Icardi and M. Di Sciuva, Large-deflection and stress analysis of multilayered plates with induced-strain actuators. Smart Mater. Struct., 5, 140-164, 1996.

[4] S. Yi, S. F. Ling and M. Ying, Large deformation finite element analyses of composite structures integrated with piezoelectric sensors and actuators. Finite Elements in Analysis and Design, 35, 1-15, 2000.

[5] A. Mukherjee and A. S. Chaudari, Piezolaminated beams with large deformations. Int. J. of Solids and Structures, 39, 4567-4582, 2002.

[6] S. Lentzen and R. Schmidt, Nonlinear finite element modelling of composite structures with integrated piezoelectric layers. High Performance Structures and Materials II, WIT Press, Southampton-Boston, 67-76, 2004. 
[7] M. R. M. Krishna, and C. Mei, Finite element buckling and post-buckling analyses of a plate with piezoelectric actuator. In Proc. of the Conference on Recent Advances in Adaptive and Sensory Materials and Their Applications, Blacksburg, USA. 301-313, 1992.

[8] K. Chandrashekhara and K. Bhatia, Active buckling control of smart composite plates - finite element analysis. Smart Mater. Struct., 2, 31-39, 1993.

[9] H. S. Tzou, Y. Bao and R. Ye, In Smart Structures and Materials 1994: Smart Structures and Intelligent Systems, Proc. of SPIE, Vol. 2190. 206-214, 1994.

[10] P. F. Pai, A. H. Nayfeh, K. Oh and D. T. Mook, A refined nonlinear model of composite plates with integrated piezoelectric actuators and sensors. Int. J. of Solids and Struct., 30, 1603-1630, 1993.

[11] R. L. Clark and S. E. Burke, Practical limitation in achieving shaped modal sensors with induced strain materials. J. Vib. Acoust., 118, 668-675, 1996.

[12] C. K. Lee and F. C. Moon, Modal sensors/actuators. J. Appl. Mech, 57, 434441, 1996.

[13] W. Gawronski, Modal actuators and sensors. J. Sound Vib., 229, 1013-1022, 2000.

[14] A. M. Sadri, J. R. Wright and R. J. Wynne, Modelling and placement of piezoelectric actuators in isotropic plates using genetic algorithms. Smart Mater. Struct., 8, 490-498, 1999.

[15] J. H. Han and I. Lee, Optimal placement of piezoelectric sensors and actuators for vibration control of a composite plate using genetic algorithms. Smart Mater. Struct., 8, 257-267, 1999.

[16] R. Schmidt and J. N. Reddy, A refined small strain and moderate rotation theory of elastic anisotropic shells. J. Appl Mech, 55, 611-617, 1988.

[17] S. Lentzen and R. Schmidt, Nonlinear FE-simulation of piezolaminated plates and shells, Proc. International Congress on Computational Mechanics \& Simulation, ICCMS-04, IIT Kanpur (India), vol. I, 77-85, 2004.

[18] A. Hac and L. Liu, Sensor actuator location in motion control of flexible structures. J. Sound Vib., 167, 239-261, 1993.

[19] W. Gawronski, 1998, Dynamics and Control of Structures: A Modal Approach, Springer.

[20] I. Bruant, G. Coffignal and F. Lene, A methodology for determination of piezoelectric actuator and sensor location on beam structures. J. Sound Vib., 243, 861-882, 2001.

[21] S. L. Padula and R. K. Kinkaid, Optimisation strategies for sensor and actuator placement. National Aeronautics and Space Administration (NASA), Langley Research Center, Langley, Virginia, 1999.

[22] D. Halim and S. O. Reza Moheimani, An optimisation approach to optimal placement of collocated piezoelectric actuators and sensors on a thin plate. Mechatronics, 13, 27-47, 2001. 